Kragujevac Journal of Mathematics

Volume 41(2) (2017), Pages 251-258.

\title{
SOLUTION OF A PARTIAL DIFFERENTIAL EQUATION RELATED TO THE OPERATOR $\oplus_{B}^{k}$
}

\author{
S. BUPASIRI ${ }^{1}$
}

Abstract. In this paper, we consider the equation

$$
\oplus_{B}^{k} u(x)=\sum_{r=o}^{m} c_{r} \oplus_{B}^{r} \delta
$$

where $\oplus_{B}^{k}$ is the operator iterated $k$-time and is defined by

$$
\oplus_{B}^{k}=\left[\left(B_{x_{1}}+B_{x_{2}}+\cdots+B_{x_{p}}\right)^{4}-\left(B_{x_{p+1}}+B_{x_{p+2}}+\cdots+B_{x_{p+q}}\right)^{4}\right]^{k},
$$

where $p+q=n, x=\left(x_{1}, \ldots, x_{n}\right) \in \mathbb{R}_{n}^{+}, B_{x_{i}}=\frac{\partial^{2}}{\partial x_{i}^{2}}+\frac{2 v_{i}}{x_{i}} \frac{\partial}{\partial x_{i}}, v_{i}=2 \alpha_{i}+1, \alpha_{i}>-\frac{1}{2}$, $x_{i}>0, i=1,2, \ldots, n, c_{r}$ is a constant, $k$ is a nonnegative integer, $\delta$ is the Dirac-delta distribution, $\oplus_{B}^{0} \delta=\delta$ and $n$ is the dimension of $\mathbb{R}_{n}^{+}$. It is shown that, depending on the relationship between $k$ and $m$, the solution to this equation can be ordinary functions, tempered distributions, or singular distributions.

\section{INTRODUCTION}

Bupasiri [5] has first introduced the elementary solution of the $n$-dimensional $\oplus_{B}^{k}$ operator and showed that the solution of the convolution form $(-1)^{3 k} S_{6 k}(x) * R_{6 k}(x) *$ $\left(C^{* k}(x)\right)^{*-1}$ is a unique elementary solution of the $\oplus_{B}^{k} u(x)=\delta$.

Key words and phrases. Bessel diamond operator, O-plus operator, Dirac-delta distribution. 2010 Mathematics Subject Classification. Primary: 46F10. Secondary: 46F20.

Received: August 7, 2016.

Accepted: October 27, 2016. 
Yildirim, Sarikaya and Ozturk [3] studied the Bessel diamond operator, iterated $k$-times,

$$
\begin{aligned}
\diamond_{B}^{k} & =\left[\left(\sum_{i=1}^{p} B_{x_{i}}\right)^{2}-\left(\sum_{j=p+1}^{p+q} B_{x_{j}}\right)^{2}\right] \\
& =\left[\sum_{i=1}^{p} B_{x_{i}}-\sum_{j=p+1}^{p+q} B_{x_{j}}\right]^{k}\left[\sum_{i=1}^{p} B_{x_{i}}+\sum_{j=p+1}^{p+q} B_{x_{j}}\right]^{k} .
\end{aligned}
$$

Yildirim, Sarikaya and Ozturk [3] showed that the function $u(x)=(-1)^{k} S_{2 k}(x) * R_{2 k}(x)$ is the unique elementary solution for the operator $\diamond_{B}^{k}$, where $*$ indicates convolution, and $R_{2 k}(x), S_{2 k}(x)$ are defined by (1.4) and (1.5) respectively, that is,

$$
\diamond_{B}^{k}\left((-1)^{k} S_{2 k}(x) * R_{2 k}(x)\right)=\delta(x) .
$$

We consider the equation

$$
\oplus_{B}^{k} u(x)=\sum_{r=o}^{m} c_{r} \oplus_{B}^{r} \delta,
$$

where $\oplus_{B}^{k}$ is the operator iterated $k$-time and is defined by

$$
\begin{aligned}
\oplus_{B}^{k} & =\left[\left(\sum_{i=1}^{p} B_{x_{i}}\right)^{4}-\left(\sum_{j=p+1}^{p+q} B_{x_{j}}\right)^{4}\right]^{k} \\
& =\left[\left(\sum_{i=1}^{p} B_{x_{i}}\right)^{2}-\left(\sum_{j=p+1}^{p+q} B_{x_{j}}\right)^{2}\right]^{k}\left[\left(\sum_{i=1}^{p} B_{x_{i}}\right)^{2}+\left(\sum_{j=p+1}^{p+q} B_{x_{j}}\right)^{2}\right]^{k} \\
& =\diamond_{B}^{k} \odot_{B}^{k},
\end{aligned}
$$

where

$$
\begin{aligned}
\odot_{B}^{k} & =\left[\left(\sum_{i=1}^{p} B_{x_{i}}\right)^{2}+\left(\sum_{j=p+1}^{p+q} B_{x_{j}}\right)^{2}\right]^{k} \\
& =\left[\left(\frac{\triangle_{B}+\square_{B}}{2}\right)^{2}+\left(\frac{\triangle_{B}-\square_{B}}{2}\right)^{2}\right]^{k} \\
& =\left(\frac{\triangle_{B}^{2}+\square_{B}^{2}}{2}\right)^{k} .
\end{aligned}
$$

The purpose of this article, is finding the solution to the equation

$$
\oplus_{B}^{k} u(x)=\sum_{r=0}^{m} c_{r} \oplus_{B}^{r} \delta
$$


by using convolutions of the generalized function. It is also shown that the type of solution to (1.3) depends on the relationship between $k$ and $m$, according to the following cases:

(1) If $m<k$ and $m=0$, then (1.3) has the solution

$$
u(x)=c_{0}\left((-1)^{3 k} S_{6 k}(x) * R_{6 k}(x) *\left(C^{* k}(x)\right)^{*-1}\right),
$$

which is an elementary solution of the $\oplus_{B}^{k}$ operator in Theorem 2.2, is an ordinary function when $6 k \geq n$, and is a tempered distribution when $6 k<n$.

(2) If $m<k$ then the solution of (1.3) is

$$
u(x)=\sum_{r=1}^{m} c_{r} c_{0}\left((-1)^{3(k-r)} S_{6(k-r)}(x) * R_{6(k-r)}(x) *\left(C^{*(k-r)}(x)\right)^{*-1}\right),
$$

which is an ordinary function when $6 k-6 r \geq n$ and is tempered distribution when $6 k-6 r<n$.

(3) If $m \geq k$ and $k \leq m \leq M$, then (1.3) has the solution

$$
u(x)=\sum_{r=k}^{M} c_{r} \oplus_{B}^{r-k} \delta,
$$

which is only a singular distribution. Before going that point, the following definitions and some concepts are needed.

Lemma 1.1. Given the equation $\square_{B}^{k} u(x)=\delta(x)$ for $x \in \mathbb{R}_{n}^{+}=\left\{x: x=\left(x_{1}, \cdots x_{n}\right)\right.$, $\left.x_{1}>0, \ldots, x_{n}>0\right\}$, where $\square_{B}^{k}$ is the Bessel-ultra hyperbolic operator iterated $k$-times. Then $u(x)=R_{2 k}(x)$ is an elementary solution of the operator $\square_{B}^{k}$, where

$$
\begin{aligned}
\square_{B}^{k} & =\left[\sum_{i=1}^{p} B_{x_{i}}-\sum_{j=p+1}^{p+q} B_{x_{j}}\right]^{k}, \\
R_{2 k}(x) & =\frac{V^{\frac{2 k-n-|v|}{2}}}{K_{n}(2 k)} \\
& =\frac{\left(x_{1}^{2}+x_{2}^{2}+\cdots+x_{p}^{2}-x_{p+1}^{2}-x_{p+2}^{2}-\cdots-x_{p+q}^{2}\right)\left(\frac{2 k-n-|v|}{2}\right)}{K_{n}(2 k)},
\end{aligned}
$$

for

and

$$
V=x_{1}^{2}+x_{2}^{2}+\cdots+x_{p}^{2}-x_{p+1}^{2}-x_{p+2}^{2}-\cdots-x_{p+q}^{2},
$$

$$
K_{n}(2 k)=\frac{\pi^{\frac{n+2|v|-1}{2}} \Gamma\left(\frac{2+2 k-n-2|v|}{2}\right) \Gamma\left(\frac{1-2 k}{2}\right) \Gamma(2 k)}{\Gamma\left(\frac{2+2 k-p-2|v|}{2}\right) \Gamma\left(\frac{p+2|v|-2 k}{2}\right)} .
$$

Lemma 1.2. Given the equation $\triangle_{B}^{k} u(x)=\delta(x)$ for $x \in \mathbb{R}_{n}^{+}$, where $\triangle_{B}^{k}$ is the Laplace Bessel operator iterated $k$-times. Then $u(x)=(-1)^{k} S_{2 k}(x)$ is an elementary solution of the operator $\triangle_{B}^{k}$, where 


$$
\begin{aligned}
\triangle_{B}^{k} & =\left[\sum_{i=1}^{p} B_{x_{i}}+\sum_{j=p+1}^{p+q} B_{x_{j}}\right]^{k}, \\
S_{2 k}(x) & =\frac{|x|^{2 k-n-2|v|}}{w_{n}(2 k)}, \quad p+q=n, \\
|x| & =\left(x_{1}^{2}+x_{2}^{2}+\cdots+x_{n}^{2}\right)^{\frac{1}{2}},
\end{aligned}
$$

and

$$
w_{n}(2 k)=\frac{\prod_{i=1}^{n} 2^{v_{i}-\frac{1}{2}} \Gamma\left(v_{i}+\frac{1}{2}\right) \Gamma(k)}{2^{n+2|v|-4 k} \Gamma\left(\frac{n+2|v|-2 k}{2}\right)} .
$$

Lemma 1.3. The convolution $R_{2 k}(x) *(-1)^{k} S_{2 k}(x)$ is an elementary solution for the operator $\diamond_{B}^{k}$ iterated $k$-times and is defined by (1.1).

Lemma 1.4. $R_{2 k}(x)$ and $S_{2 k}(x)$ are homogeneous distributions of order $(2 k-n-2|v|)$.

We need to show that $R_{2 k}(x)$ and $(-1)^{k} S_{2 k}(x)$ satisfy the Euler equation; that is,

$$
\begin{aligned}
(2 k-n-2|v|) R_{2 k}(x) & =\sum_{i=1}^{n} x_{i} \frac{\partial}{\partial x_{i}} R_{2 k}(x), \\
(2 k-n-2|v|) S_{2 k}(x) & =\sum_{i=1}^{n} x_{i} \frac{\partial}{\partial x_{i}} S_{2 k}(x) .
\end{aligned}
$$

Lemma 1.5 (The $B$-convolution of tempered distribution). $R_{2 k}(x) * S_{2 k}(x)$ exists and is a tempered distribution.

Proof. For the proofs of Lemmas 1.1-1.5, see [3, p. 378-383].

Lemma 1.6 (The $B$-convolution of $R_{2 k}(x)$ and $S_{2 k}(x)$ ). Let $R_{2 k}(x)$ and $S_{2 k}(x)$ defined by (1.4) and (1.5) respectively, then we obtain:

(1) $S_{2 k}(x) * S_{2 m}(x)=S_{2 k+2 m}(x)$, where $k$ and $m$ are nonnegative integers.

(2) $R_{2 k}(x) * R_{2 m}(x)=R_{2 k+2 m}(x)$, where $k$ and $m$ are nonnegative integers.

Lemma 1.7. The function $R_{-2 k}(x)$ and $(-1)^{k} S_{-2 k}(x)$ are the inverse in the convolution algebra of $R_{2 k}(x)$ and $(-1)^{k} S_{2 k}(x)$, respectively. That is,

$$
\begin{aligned}
R_{-2 k}(x) * R_{2 k}(x) & =R_{-2 k+2 k}(x)=R_{0}(x)=\delta(x), \\
(-1)^{k} S_{-2 k}(x) *(-1)^{k} S_{2 k}(x) & =S_{-2 k+2 k}(x)=S_{0}(x)=\delta(x) .
\end{aligned}
$$

Proof. For the proofs of Lemma 1.7 and Lemma 1.6, see [4].

Lemma 1.8. Given the equation

$$
\oplus_{B}^{k} u(x)=\delta(x),
$$


where $\oplus_{B}^{k}$ is the operator iterated $k$-times defined by (1.2), $\delta(x)$ is the Dirac-delta distribution, $x \in \mathbb{R}_{n}^{+}$and $k$ is a nonnegative integer. Then we obtain

$$
u(x)=\left(R_{6 k}(x) *(-1)^{3 k} S_{6 k}(x)\right) *\left(C^{* k}(x)\right)^{*-1}
$$

is a Green's function or an elementary solution for the operator $\oplus_{B}^{k}$ iterated $k$-times where $\oplus_{B}^{k}$ is defined by (1.2), and

$$
C(x)=\frac{1}{2} R_{4}(x)+\frac{1}{2}(-1)^{2} S_{4}(x),
$$

where $C^{* k}(x)$ denotes the convolution of $C$ with itself $k$ times, $\left(C^{* k}(x)\right)^{*-1}$ denotes the inverse of $C^{* k}(x)$ in the convolution algebra. Moreover $u(x)$ is a tempered distribution.

Proof. For a proof of the above lemma, see [5].

\section{MAin Results}

Theorem 2.1. For $0<r<k$,

$$
\begin{aligned}
& \oplus_{B}^{k}\left(c_{0}\left((-1)^{3 k} S_{6 k}(x) * R_{6 k}(x) *\left(C^{* k}(x)\right)^{*-1}\right)\right) \\
= & \left((-1)^{3(k-r)} S_{6(k-r)}(x) * R_{6(k-r)}(x) *\left(C^{*(k-r)}(x)\right)^{*-1}\right)
\end{aligned}
$$

and for $k \leq m$,

$$
\oplus_{B}^{m}\left(c_{0}\left((-1)^{3 k} S_{6 k}(x) * R_{6 k}(x) *\left(C^{* k}(x)\right)^{*-1}\right)\right)=\oplus_{B}^{m-k} \delta .
$$

Proof. For $0<r<k$, from (1.6),

$$
\oplus_{B}^{k}\left(c_{0}\left((-1)^{3 k} S_{6 k}(x) * R_{6 k}(x) *\left(C^{* k}(x)\right)^{*-1}\right)=\delta .\right.
$$

Thus,

$$
\oplus_{B}^{k-r} \oplus_{B}^{r}\left(c_{0}\left((-1)^{3 k} S_{6 k}(x) * R_{6 k}(x) *\left(C^{* k}(x)\right)^{*-1}\right)=\delta\right.
$$

or

$$
\oplus_{B}^{k-r} \delta * \oplus_{B}^{r}\left(c_{0}\left((-1)^{3 k} S_{6 k}(x) * R_{6 k}(x) *\left(C^{* k}(x)\right)^{*-1}\right)=\delta .\right.
$$

Convolving both sides by $\left((-1)^{3(k-r)} S_{6(k-r)}(x) * R_{6(k-r)}(x) *\left(C^{*(k-r)}(x)\right)^{*-1}\right)$, we obtain

$$
\begin{aligned}
& \oplus_{B}^{k-r}\left(\left((-1)^{3(k-r)} S_{6(k-r)}(x) * R_{6(k-r)}(x) *\left(C^{*(k-r)}(x)\right)^{*-1}\right)\right) \\
& * \oplus_{B}^{r}\left(c_{0}\left((-1)^{3 k} S_{6 k}(x) * R_{6 k}(x) *\left(C^{* k}(x)\right)^{*-1}\right)\right. \\
= & \left((-1)^{3(k-r)} S_{6(k-r)}(x) * R_{6(k-r)}(x) *\left(C^{*(k-r)}(x)\right)^{*-1}\right) * \delta .
\end{aligned}
$$

By Lemma 1.8,

$$
\begin{aligned}
& \delta * \oplus_{B}^{r}\left(c_{0}\left((-1)^{3 k} S_{6 k}(x) * R_{6 k}(x) *\left(C^{* k}(x)\right)^{*-1}\right)\right. \\
= & \left((-1)^{3(k-r)} S_{6(k-r)}(x) * R_{6(k-r)}(x) *\left(C^{*(k-r)}(x)\right)^{*-1}\right) * \delta .
\end{aligned}
$$

It follows that

$$
\begin{aligned}
& \oplus_{B}^{r}\left(c_{0}\left((-1)^{3 k} S_{6 k}(x) * R_{6 k}(x) *\left(C^{* k}(x)\right)^{*-1}\right)\right. \\
= & \left((-1)^{3(k-r)} S_{6(k-r)}(x) * R_{6(k-r)}(x) *\left(C^{*(k-r)}(x)\right)^{*-1}\right),
\end{aligned}
$$


as required. For $k \leq m$

$$
\begin{aligned}
& \oplus_{B}^{m}\left(c_{0}\left((-1)^{3 k} S_{6 k}(x) * R_{6 k}(x) *\left(C^{* k}(x)\right)^{*-1}\right)\right. \\
= & \oplus_{B}^{m-k} \oplus_{B}^{k}\left((-1)^{3 k} S_{6 k}(x) * R_{6 k}(x) *\left(C^{* k}(x)\right)^{*-1}\right) .
\end{aligned}
$$

It follows that

$$
\oplus_{B}^{m}\left(c_{0}\left((-1)^{3 k} S_{6 k}(x) * R_{6 k}(x) *\left(C^{* k}(x)\right)^{*-1}\right)=\oplus_{B}^{m-k} \delta\right.
$$

by Lemma 1.8. This completes the proof.

Theorem 2.2. Consider the linear differential equation

$$
\oplus_{B}^{k} u(x)=\sum_{r=0}^{m} c_{r} \oplus_{B}^{r} \delta
$$

where $p+q=n, n$ is odd with $p$ odd and $q$ even, or $n$ is even with $p$ odd and $q$ odd, $x \in \mathbb{R}_{n}^{+}=\left\{x: x=\left(x_{1}, \ldots, x_{n}\right), x_{1}>0, \ldots, x_{n}>0\right\}, c_{r}$ is a constant, $\delta$ is the Dirac-delta distribution, and $\oplus_{B}^{0} \delta=\delta$. Then the type of solution to (2.1) depends on the relationship between $k$ and $m$, according to the following cases:

(1) If $m<k$ and $m=0$, then (2.1) has the solution

$$
u(x)=c_{0}\left((-1)^{3 k} S_{6 k}(x) * R_{6 k}(x) *\left(C^{* k}(x)\right)^{*-1}\right),
$$

which is an elementary solution of the $\oplus_{B}^{k}$ operator in Theorem 2.1, is an ordinary function when $6 k \geq n$, and is a temper distribution when $6 k<n$.

(2) If $m<k$ then the solution of (2.1) is

$$
u(x)=\sum_{r=1}^{m} c_{r}\left((-1)^{3(k-r)} S_{6(k-r)}(x) * R_{6(k-r)}(x) *\left(C^{*(k-r)}(x)\right)^{*-1}\right),
$$

which is an ordinary function when $6 k-6 r \geq n$ and is tempered distribution when $6 k-6 r<n$.

(3) If $m \geq k$ and $k \leq m \leq M$, then (2.1) has the solution

$$
u(x)=\sum_{r=k}^{M} c_{r} \oplus_{B}^{r-k} \delta,
$$

which is only a singular distribution.

Proof. (1) For $m=0$, we have $\oplus_{B}^{k} u(x)=c_{0} \delta$, and by Theorem 2.1 we obtain

$$
u(x)=\left((-1)^{3 k} S_{6 k}(x) * R_{6 k}(x) *\left(C^{* k}(x)\right)^{*-1}\right) .
$$

Now, $(-1)^{3 k} S_{6 k}(x)$ and $R_{6 k}(x)$ are the analytic function for $6 k \geq n$ and also $(-1)^{3 k} S_{6 k}(x) * R_{6 k}(x) *\left(C^{* k}(x)\right)^{*-1}$ exits and is an analytic function by (1.7). It follows that $(-1)^{3 k} S_{6 k}(x) * R_{6 k}(x) *\left(C^{* k}(x)\right)^{*-1}$ is an ordinary function for $6 k \geq n$. By Lemma $1.5,(-1)^{3 k} S_{6 k}(x), R_{6 k}(x)$ are tempered distributions with $6 k<n$, we obtain $(-1)^{3 k} S_{6 k}(x) * R_{6 k}(x) *\left(C^{* k}(x)\right)^{*-1}$ exits and is a tempered distribution. 
(2) For the case $0<m<k$, we have

$$
\oplus_{B}^{k} u(x)=c_{1} \oplus_{B} \delta+c_{2} \oplus_{B}^{2} \delta+\cdots+c_{m} \oplus_{B}^{m} \delta .
$$

We convolved both sides of the above equation by $(-1)^{3 k} S_{6 k}(x) * R_{6 k}(x) *$ $\left(C^{* k}(x)\right)^{*-1}$ to obtain

$$
\begin{aligned}
& \oplus_{B}^{k}\left((-1)^{3 k} S_{6 k}(x) * R_{6 k}(x) *\left(C^{* k}(x)\right)^{*-1}\right) * u(x) \\
= & c_{1} \oplus_{B}\left((-1)^{3 k} S_{6 k}(x) * R_{6 k}(x) *\left(C^{* k}(x)\right)^{*-1}\right) \\
& +c_{2} \oplus_{B}^{2}\left((-1)^{3 k} S_{6 k}(x) * R_{6 k}(x) *\left(C^{* k}(x)\right)^{*-1}\right) \\
& +\cdots+c_{m} \oplus_{B}^{m}\left((-1)^{3 k} S_{6 k}(x) * R_{6 k}(x) *\left(C^{* k}(x)\right)^{*-1}\right) .
\end{aligned}
$$

By Theorem 2.1, we obtain

$$
\begin{aligned}
u(x)= & c_{1}\left((-1)^{3(k-1)} S_{6(k-1)}(x) * R_{6(k-1)}(x) *\left(C^{*(k-1)}(x)\right)^{*-1}\right) \\
& +c_{2}\left((-1)^{4(k-2)} S_{6(k-2)}(x) * R_{6(k-2)}(x) *\left(C^{*(k-2)}(x)\right)^{*-1}\right) \\
& +\cdots+c_{m}\left((-1)^{3(k-m)} S_{6(k-m)}(x) * R_{6(k-m)}(x) *\left(C^{*(k-m)}(x)\right)^{*-1}\right),
\end{aligned}
$$

or

$$
u(x)=\sum_{r=1}^{m} c_{r}\left((-1)^{3(k-r)} S_{6(k-r)}(x) * R_{6(k-r)}(x) *\left(C^{*(k-r)}(x)\right)^{*-1}\right) .
$$

Similarly, as in case (1), $u(x)$ is an ordinary function for $6 k-6 r \geq n$ and is a tempered distribution for and $6 k-6 r<n$.

(3) For the case $m \geq k$ and $k \leq m \leq M$, we have

$$
\oplus_{B}^{k} u(x)=c_{k} \oplus_{B}^{k} \delta+c_{k+1} \oplus_{B}^{k+1} \delta+\cdots+c_{M} \oplus_{B}^{M} \delta .
$$

Convolved both sides of the above equation by

$$
(-1)^{3 k} S_{6 k}(x) * R_{6 k}(x) *\left(C^{* k}(x)\right)^{*-1}
$$

to obtain

$$
\begin{aligned}
& \oplus_{B}^{k}\left((-1)^{3 k} S_{6 k}(x) * R_{6 k}(x) *\left(C^{* k}(x)\right)^{*-1}\right) * u(x) \\
= & c_{k} \oplus_{B}^{k}\left((-1)^{3 k} S_{6 k}(x) * R_{6 k}(x) *\left(C^{* k}(x)\right)^{*-1}\right) \\
& +c_{k+1} \oplus_{B}^{k+1}\left((-1)^{3 k} S_{6 k}(x) * R_{6 k}(x) *\left(C^{* k}(x)\right)^{*-1}\right) \\
& +\cdots+c_{M} \oplus_{B}^{M}\left((-1)^{3 k} S_{6 k}(x) * R_{6 k}(x) *\left(C^{* k}(x)\right)^{*-1}\right) .
\end{aligned}
$$

By Theorem 2.1 again, we obtain

$$
u(x)=c_{k} \delta+c_{k+1} \oplus_{B} \delta+c_{k+2} \oplus_{B}^{2} \delta+\cdots+c_{M} \oplus_{B}^{M-k} \delta=\sum_{r=k}^{M} c_{r} \oplus_{B}^{r-k} \delta .
$$

Since $\oplus_{B}^{r-k} \delta$ is a singular distribution, hence $u(x)$ is only the singular distribution. This completes the proofs. 
Acknowledgements. The author would like to thank the referees for their suggestions which enhanced the presentation of the paper. The author was supported by Sakon Nakhon Rajabhat University.

\section{REFERENCES}

[1] A. H. Zemanian, Distribution and Transform Analysis, McGraw-Hill, New York, 1965.

[2] B. M. Levitan, Expansion in Fourier series and integrals with Bessel functions, Uspekhi Mat. Nauk 6(42), (1951), 102-143.

[3] H. Yildirim, M. Z. Sarikaya and S. Ozturk, The solutions of the n-dimensional Bessel diamond operator and the Fourier-Bessel transform of their convolution, Proc. Indian Acad. Sci. Math. Sci. 114(4) (2004), 375-387.

[4] M. Z. Sarikaya and H. Yildirim, On the B-convolutions of the Bessel diamond kernel of Riesz, Appl. Math. Comput. 208 (2009), 18-22.

[5] S. Bupasiri, On the solution of the $n$-dimensional $\oplus_{B}^{k}$ operator, Applied Mathematical Sciences 9(10), (2015), 469-479.

[6] W. Satsanit, Green function and Fourier transform for o-plus operator, Electron. J. Differential Equations 2010 (2010), Article ID: 48.

${ }^{1}$ FACULTY OF EDUCATION

SAKON NAKHON RAJABHAT UnIVERSITY

THAILAND 47000

E-mail address: sudprathai@gmail.com 